\title{
Role of progenitor cell marker CD133 in supporting diagnosis of collapsing glomerulopathy
}

\author{
Andrew Y. Xiao ${ }^{1}$ (1) $\cdot$ Hassan D. Kanaan ${ }^{2} \cdot{\text { Zongshan } \mathrm{Lai}^{2} \cdot \mathrm{Wei}^{\mathrm{Li}}{ }^{2} \cdot \text { Ping L. Zhang }}^{2}$
}

Received: 21 April 2021 / Accepted: 22 November 2021 / Published online: 3 January 2022

(c) The Author(s), under exclusive licence to Springer Nature B.V. 2021

\begin{abstract}
Purpose A previous immunofluorescent study suggests that, in collapsing glomerulopathy, most hyperplastic podocytes that stained positively for a progenitor cell marker CD133 are derived from CD133 + parietal epithelial cells. In pathology practice, not all renal biopsies with collapsing glomerulopathy show the typical morphologic features for this entity, which include florid podocyte hyperplasia, collapsing glomerular capillary loops, and cystic tubular dilation. This study was made to determine if CD133 staining using an immunohistochemical method can be used to confirm hyperplastic podocytes and identify extensive acute tubular injury in collapsing glomerulopathy.

Methods Twenty-one collapsing glomerulopathy biopsies were stained for CD133 and compared with 15 biopsies with focal segmental glomerulosclerosis, not otherwise specified (FSGS).

Results All patients with collapsing glomerulopathy were of African American descent with prominent renal failure and nephrotic range proteinuria. In contrast, the FSGS group consisted of patients from a variety of ethnic backgrounds with nephrotic range proteinuria but relatively low serum creatinine. The striking finding was that all collapsing glomerulopathy cases showed positive CD133 staining in the clusters of hyperplastic podocytes. There was significantly higher CD133positive staining rate for hyperplastic podocytes $(38 \%)$ in the glomeruli of the collapsing glomerulopathy group when compared to small clusters of hyperplastic podocytes in the FSGS group (8\%). In addition, when compared to the relatively weak CD133 staining in the proximal tubules of the FSGS group, the proximal tubules of the collapsing glomerulopathy group all showed diffuse and strong CD133 staining as a feature of severe acute tubular injury, which corresponded to the high serum creatinine levels in these patients.
\end{abstract}

Conclusion Our data indicate that the combination of the distinctive mosaic CD133 staining in hyperplastic podocytes and the diffuse tubular CD133 staining is helpful in supporting a diagnosis of collapsing glomerulopathy.

Keywords Collapsing glomerulopathy $\cdot$ Focal segmental glomerulosclerosis $\cdot$ CD133 $\cdot$ Progenitor cells $\cdot$ Parietal epithelium $\cdot$ Proliferative podocytes $\cdot$ Acute tubular injury

\section{Introduction}

CD133, also known as prominin-1, is a membranous glycoprotein encoded by the PROM1 gene in human, but its specific functions remain unclear $[1,2]$. CD133 has been investigated for its potential utility as a stem cell marker in different adult organs and tumors [2]. The activation of

Ping L. Zhang

Ping.Zhang@Beaumont.edu

1 William Beaumont School of Medicine, Oakland University, Rochester, MI, USA

2 Beaumont Labs, Division of Anatomic Pathology, Beaumont Health, Royal Oak, MI, USA
CD133 in the stem cells of the kidney may be related to the activation of hypoxia induced factors (HIFs) and their master mechanism for oxygen sensing and gene regulations $[3,4]$. The parietal epithelium along the Bowman's capsule stains positively for the progenitor markers CD133 and CD24 and are thus identified as multipotent progenitor cells [5-7]. The parietal epithelium continuously differentiates into the visceral epithelium (podocytes) of glomeruli, which are terminally differentiated and thus stain negatively for CD133. Some tracing studies also support the view that podocytes are derived from the parietal epithelium [8,9]. In addition, the CD133-positive progenitor cells are also scattered along proximal tubules under normal circumstances [10-12]. The injection of CD133 progenitor cells into variants of rodent 
models with acute renal failure and/or proteinuria can significantly improve the renal function and reduce proteinuria, implying the regenerative capacity of the renal progenitor cells $[6,7]$.

Diagnosis of collapsing glomerulopathy can be challenging if the typical features, such as florid podocyte hyperplasia and obvious cystic dilation in renal tubules, are absent. Collapsing glomerulopathy (also known as collapsing variant of focal segmental glomerulosclerosis) can result from multiple etiologies, including HIV infection and genetic abnormalities such as APOL1 mutations in African American patients [13, 14]. Recently, there are scattered reports of collapsing glomerulopathy in African American patients that contracted COVID-19 infection, but whether the collapsing glomerulopathy is directly related to the viral infection remains debatable [15-17].

Regarding the origin of the hyperplastic podocytes in the collapsing glomerulopathy, one key study using immunofluorescent techniques indicates that cellular crescents in crescentic glomerulonephritis and hyperplastic podocytes in collapsing glomerulopathy all come from the same parietal epithelium of the glomeruli [18]. The study also finds that there are 3 populations of hyperplastic podocytes in the collapsing glomerulopathy. Using confocal microscopy to evaluate several merged marker expressions, the study determined that there are approximately $60 \%$ immature podocytes $\left(\mathrm{CD} 133^{+}, \mathrm{CD}^{2} 4^{+}\right.$, podocalyxin ${ }^{-}$[PDX, a podocyte marker] nestin $^{-}$[another podocyte marker]), 30\% mature podocytes $\left(\mathrm{CD}_{133^{-}}, \mathrm{CD}^{2} 4^{-}, \mathrm{PDX}^{+}\right.$, nestin $\left.{ }^{+}\right)$, and $10 \%$ transitional podocytes $\left(\mathrm{CD} 133^{+}, \mathrm{CD} 24^{+}, \mathrm{PDX}^{+}\right.$, nestin $\left.{ }^{+}\right)$[18]. The finding indicates that glomerular hyperplastic lesions are derived from the proliferation of renal progenitors at different stages of their differentiation toward mature podocytes [18].

In this study, we first investigated whether immunohistochemical staining for CD133 can be practically used to confirm the majority of hyperplastic podocytes [18] for supporting a diagnosis of collapsing glomerulopathy. Secondly, we hypothesized that the immuno-staining for CD133 can be used to highlight the extensive acute tubular injury of collapsing glomerulopathy. We found that CD133 staining identified a mosaic pattern of hyperplastic podocytes intermingled with collapsed glomerular loops and was also diffusely positive in the injured proximal tubules. These dual CD133 staining features are helpful in supporting a diagnosis of collapsing glomerulopathy.

\section{Methods}

\section{Collecting groups of cases}

The retrospective human investigation protocol was approved by the Institutional Research Board of Beaumont
Health, Michigan. In our study, there were 3 groups of renal cases. The first group consisted of negative controls (group $1, n=12$ ) that were taken from a de-identified archive of normal renal parenchyma (either biopsies or nephrectomy specimens for renal tumors). Biopsies with primary FSGS in the second group and collapsing glomerulopathy in the third group were used for comparison as, in adults, both FSGS and collapsing glomerulopathy represent a spectrum of podocytopathies [19]. The second group was composed of 15 renal biopsies with FSGS. Our primary FSGS biopsies were pathologically characterized with at least one glomerulus with focal segmental sclerosis by light microscopy, negative immunofluorescent studies, and diffuse fusion of foot processes in electron microscopy. In the third group, we identified 21 cases of collapsing glomerulopathy with available tissue blocks over the past 10 years. The collapsing glomerulopathy biopsies were pathologically defined with at least one glomerulus showing collapsed loops, hyperpleastic podocytes, mild to severe cystic dilation of renal tubules, negative immunofluorescent studies, and diffuse fusion of foot processes by electron microscopy. Relevant clinical information for each patient was recorded through chart reviews, which included age, gender, ethnic background, serum creatinine $(\mathrm{sCr})$ levels, and protein/creatinine ratio before renal biopsies.

\section{Kidney histology and immunohistochemical assessment of CD133 expression}

Human kidney biopsy cores were fixed in formalin- and paraffin-embedded. Four-micron sections were dried for $60 \mathrm{~min}$ at $60{ }^{\circ} \mathrm{C}$. Slides were then de-waxed in 3 xylene baths for 3 min each, $100 \%$ alcohol baths for 3 min each, and then followed by $30 \mathrm{~s}$ of running water. Antigen retrieval was carried out in a Tris-EDTA Buffer at $\mathrm{pH} 8.0$ for $20 \mathrm{~min}$ at $99^{\circ} \mathrm{C}$, followed by a 20 -min cool down at room temperature, and then a quick water rinse. Slides were then placed in $3 \%$ $\mathrm{H}_{2} \mathrm{O}_{2}$ for 15 min followed by a quick water rinse and then placed in Tris buffer at $\mathrm{pH}$ 7.6. Slides were then stained in a programmed Dako Autostainer (DakoCytomation, Carpinteria, CA) using a Thermo Scientific UltraVision LP Detection System (Kalamazoo, MI). The program consisted of $5 \mathrm{~min}$ of Ultra $\mathrm{V}$ block, 30 min of incubation with a mouse monoclonal CD133 antibody (13A4 clone at a dilution of 1:100; from ThermoFisher, Waltham, MA), with 8 min of Primary Antibody Enhancer, 10 min of HRP polymer (equivalent to secondary antibody), and $5 \mathrm{~min}$ of the chromagen DAB to achieve a brown CD133 stain. Ki-67 antibodoy (MIB-1 clone, concentrate 1:100 prekit) and WT-1 antibody (clone $6 \mathrm{~F}-\mathrm{H} 2$, predilute) were purchased from DakoCytomation (Carpinteria, CA) and Cell Marque Cooperation (Rochlin, CA), respectively. Their immune stains followed similar procedures as CD133. 


\section{PAS staining and dual staining of PAS on top of CD133 staining}

De-paraffined sections were stained for PAS using Dako Artisan PAS Staining Kit (DakoCytomation, Carpinteria, CA). The steps included $12 \mathrm{~min}$ in periodic acid solution, two washes, $12 \mathrm{~min}$ of Schiff's reagent, 5 washes, $12 \mathrm{~min}$ in Meyers's hematoxylin solution, 5 washes, and $12 \mathrm{~min}$ in blurring agent before applying a cover slip. On top of CD133 stained sections, a dual-PAS staining was achieved by following the above PAS staining procedures, to highlight glomerular basement membranes and the brush borders in proximal tubules.

\section{Quantitation of immunohistochemical staining of CD133}

CD133 staining intensity scores in glomeruli and proximal tubules were graded from 0 to $3+(0$, no staining; $1+$, weak fine granular staining along the complete luminal surface; $2+$, moderate granular staining; and $3+$, strong large granular staining). The percent distribution of CD133 expression in proximal tubules was also recorded. Using a $\mathrm{H}$-score method, the combined scores of positive CD133 in glomeruli and the combined scores of CD133 expression in proximal tubules were calculated by CD133 staining intensity multiplying CD133 +\% in glomeruli and CD133 intensity multiplying CD133+\% distribution along the proximal tubules, respectively.

\section{Statistics}

Results were expressed as the mean \pm standard error of the mean (SEM). Data of three groups were compared using one-way analysis of variants (ANOVA) and an unpaired
Student's $\mathrm{t}$ test was used for comparing the two groups (StatView program) (Table 1). A receiver operating characteristic (ROC) curve was generated using SAS 9.4 (Cary, NC, USA) to compare sensitivity and specificity across a range of sCr levels for the ability of $\mathrm{sCr}$ values to predict $\mathrm{CD} 133$ score. A $p$ value of less than 0.05 is considered statistically significant.

\section{Results}

\section{General clinical indices}

The mean age for the negative controls was approximately 9 years older than the mean age for the FSGS group, although no statistical significance was reached between the two groups (Table 1). The mean age of the collapsing glomerulopathy group was more than 13 years younger than the FSGS group and the age difference was statistically significant. Furthermore, the mean age of the negative controls was significantly older than the mean value of the collapsing glomerulopathy group $(p=0.0004)$. All three groups were composed of a mix of female and male patients. The race data for the negative controls were not available as they were de-identified cases. Patients with FSGS consisted of 3 African Americans, 11 Caucasians, and 1 Latino American, whereas patients with collapsing glomerulopathy were all of African American decent. In the collapsing glomerulopathy group, 5 patients $(5 / 21$, $19 \%$ ) had a viral infectious etiology (4 were infected by human immunodeficiency virus [HIV] and 1 had coronavirus disease 19 [COVID-19]) (Table 2), whereas the remaining patients did not have a medical history of infection $(17 / 21,81 \%)$. No viral inclusions were identified in either HIV or COVID-19 biopsies by electron microscopy.
Table 1 Clinical and pathology analysis in negative controls, FSGS, and collapsing glomerulopathy

\begin{tabular}{llll}
\hline & Negative controls & FSGS & CG \\
\hline$N$ & 12 & 15 & 21 \\
Age (years) & $64 \pm 4$ & $55 \pm 5$ & $42 \pm 4^{*}$ \\
Gender & $8 \mathrm{~F}, 4 \mathrm{M}$ & $11 \mathrm{~F}, 4 \mathrm{M}$ & $11 \mathrm{~F}, 10 \mathrm{M}$ \\
Ethnic origins & $\mathrm{NA}$ & $3 \mathrm{AA}, 11 \mathrm{C}, 1 \mathrm{~L}$ & $21 \mathrm{AA}$ \\
Serum creatinine $(\mathrm{mg} / \mathrm{dl})$ & $0.95 \pm 0.12$ & $1.57 \pm 0.27$ & $5.15 \pm 0.45^{* \#}$ \\
Protein/creatinine ratio & $\mathrm{NA}$ & $6.46 \pm 1.20$ & $10.73 \pm 1.21 \#$ \\
CD133 combined scores in PT & $0.83 \pm 0.83$ & $56.67 \pm 20.32$ & $252.38 \pm 13.92^{* \#}$ \\
CD133 + podocytes $\%$ in G & $0.00 \pm 0.00$ & $8.98 \pm 3.50$ & $34.80 \pm 4.31^{* \#}$ \\
CD133 + combined scores in G & $0.00 \pm 0.00$ & $15.43 \pm 5.78$ & $93.31 \pm 14.11^{* \#}$ \\
\hline
\end{tabular}

Data of three groups, including age, serum creatinine levels, CD133 expression in proximal tubules and glomeruli, were compared using one-way analysis of variants (ANOVA) and an unpaired Student's $t$ test was used for two group comparison, as for protein/creatinine ratio

$F$ female, $M$ male, $A A$ African American, $C$ Caucasian, $L$ Latino American, $P T$ proximal tubules, $N A$ not available, $G$ glomeruli, $F S G S$ focal segmental glomerulosclerosis, $C G$ collapsing glomerulopathy

$* p<0.05$ vs negative controls. \#p $<0.05$ vs FSGS 
Table 2 Clinical and pathologic indices in patients with FSGS, NOS and collapsing FSGS

\begin{tabular}{|c|c|c|c|c|c|}
\hline & Age/gender & $\mathrm{sCr}(\mathrm{mg} / \mathrm{dl})$ & Proteinuria & $\begin{array}{l}\text { CD133 in PT intensity, } \\
\% \text { (combine scores) }\end{array}$ & CD133 in glomeruli \\
\hline \multicolumn{6}{|c|}{ FSGS, NOS } \\
\hline 1 & $62 \mathrm{M}, \mathrm{C}$ & 4.12 & 15.1 & $2+, 10 \%(20)$ & $1+, 1 / 8(12.5 \%)$ \\
\hline 2 & $68 \mathrm{M}, \mathrm{C}$ & 0.81 & 3.7 & $1+, 20 \%(20)$ & $3+, 1 / 12(8.3 \%)$ \\
\hline 3 & $78 \mathrm{M}, \mathrm{C}$ & 1.35 & 1.8 & $1+, 20 \%(20)$ & $0,0 / 14(7.1 \%)$ \\
\hline 4 & $69 \mathrm{~F}, \mathrm{C}$ & 0.65 & 4.1 & $2+, 80 \%(160)$ & $0,0 / 4(0 \%)$ \\
\hline 5 & $66 \mathrm{~F}, \mathrm{C}$ & 0.82 & 2.9 & $0,0 \%(0)$ & $1+, 1 / 2(50 \%)$ \\
\hline 6 & $24 \mathrm{~F}, \mathrm{C}$ & 0.54 & 3.5 & $0,0 \%(0)$ & $0,0 / 8(0 \%)$ \\
\hline 7 & $32 \mathrm{~F}, \mathrm{C}$ & 0.55 & 9.4 & $0,0 \%(0)$ & $2+, 2 / 17(11 \%)$ \\
\hline 8 & $70 \mathrm{~F}, \mathrm{C}$ & 2.02 & 2.9 & $2+, 80 \%(160)$ & $0,0 / 14(0 \%)$ \\
\hline 9 & $20 \mathrm{~F}, \mathrm{AA}$ & 3.00 & 6.3 & $3+, 50 \%(150)$ & $3+, 1 / 18(5.5 \%)$ \\
\hline 10 & $38 \mathrm{~F}, \mathrm{~L}$ & 0.58 & 4.1 & $0,0 \%(0)$ & $0,0 / 28(0 \%)$ \\
\hline 11 & $65 \mathrm{~F}, \mathrm{AA}$ & 1.85 & 11.8 & $0,0 \%(0)$ & $0,0 / 14(0 \%)$ \\
\hline 12 & $46 \mathrm{~F}, \mathrm{C}$ & 1.75 & 3.7 & $2+, 20 \%(40)$ & $2+, 2 / 13(15.3 \%)$ \\
\hline 13 & $63 \mathrm{~F}, \mathrm{C}$ & 1.12 & 6.9 & $1+, 20 \%(20)$ & $0,0 / 7(0 \%)$ \\
\hline 14 & $42 \mathrm{~F}, \mathrm{C}$ & 1.92 & 16.6 & $3+, 80 \%(240)$ & $3,1 / 4(25 \%)$ \\
\hline 15 & $55 \mathrm{M}, \mathrm{AA}$ & 2.50 & 4.1 & $1+, 20 \%(20)$ & $0,0 / 7(0 \%)$ \\
\hline \multicolumn{6}{|c|}{ Collapsing FSGS } \\
\hline 1 & $51 \mathrm{~F}, \mathrm{AA}$ & 6.52 & 8.3 & $2+, 60 \%(160)$ & $2+, 6 / 15(40 \%)$ \\
\hline 2 & $53 \mathrm{~F}, \mathrm{AA}$ & 6.71 & 18.2 & $3+, 100 \%(300)$ & $3+, 1 / 6(16.6 \%)$ \\
\hline 3 & $20 \mathrm{~F}, \mathrm{AA}$ & 6.52 & 5.6 & $3+, 100 \%(300)$ & $2+, 6 / 17(35.3 \%)$ \\
\hline 4 & $48 \mathrm{~F}, \mathrm{AA}$ & 6.43 & 12.9 & $2+100 \%(200)$ & $3+, 2 / 3(60 \%)$ \\
\hline 5 & $48 \mathrm{~F}, \mathrm{AA}$ & 6.33 & 14.9 & $3+, 100 \%(300)$ & $3+, 5 / 11(45.5 \%)$ \\
\hline 6 & $29 \mathrm{~F}, \mathrm{AA}$ & 1.48 & 11.0 & $3+, 40 \%(120)$ & $2+, 1 / 13(7.6 \%)$ \\
\hline 7 & $20 \mathrm{~F}, \mathrm{AA}$ & 7.00 & 5.7 & $3+, 80 \%(240)$ & $3+, 2 / 5(40 \%)$ \\
\hline 8 & $25 \mathrm{~F}, \mathrm{AA}$ & 3.70 & 28.0 & $3+, 70 \%(210)$ & $3+, 3 / 10(30 \%)$ \\
\hline 9 & $61 \mathrm{~F}, \mathrm{AA}$ & 6.13 & 13.4 & $2+, 100 \%(200)$ & $2+, 1 / 3(33.3 \%)$ \\
\hline 10 & $45 \mathrm{~F}, \mathrm{AA}$ & 7.21 & 11.1 & $3+, 100 \%(300)$ & $2+, 1 / 3(33.3 \%)$ \\
\hline 11 & $83 \mathrm{M}, \mathrm{AA}$ & 3.80 & 13.2 & $3+, 100 \%(300)$ & $3+, 9 / 11(81.8 \%)$ \\
\hline 12 & $29 \mathrm{M}, \mathrm{AA}$ & 4.06 & 12.7 & $3+, 100 \%(300)$ & $3+3 / 14(21.4 \%)$ \\
\hline 13 & $66 \mathrm{M}, \mathrm{AA}$ & 3.40 & 5.7 & $3+, 100 \%(300)$ & $3+, 2 / 7(28.6 \%)$ \\
\hline 14 & $32 \mathrm{M} \mathrm{AA}$ & 2.43 & 7.2 & $3+, 70 \%(210)$ & $2+, 5 / 20(25 \%)$ \\
\hline 15 & $32 \mathrm{M}, \mathrm{AA}$ & 3.93 & 12.5 & $3+, 100 \%(300)$ & $3+, 2 / 3(66.7 \%)$ \\
\hline 16 & $50 \mathrm{~F}, \mathrm{AA}$ & 5.70 & 6.4 & $2+, 80 \%(160)$ & $2+, 2 / 13(15.4 \%)$ \\
\hline 17 & $* 29 \mathrm{M}, \mathrm{AA}$ & 3.20 & 5.6 & $3+, 80 \%(240)$ & $1+, 1 / 7(14.3 \%)$ \\
\hline 18 & $* 49 \mathrm{M}$ & 4.03 & 7.6 & $3+, 100 \%(300)$ & $2+, 2 / 14(14.3 \%)$ \\
\hline 19 & $* 27 \mathrm{M}, \mathrm{AA}$ & 8.21 & 14.1 & $3+, 100 \%(300)$ & $3+, 2 / 8(25 \%)$ \\
\hline 20 & $* 53 \mathrm{M}, \mathrm{AA}$ & 2.38 & 6.2 & $3+, 100 \%(300)$ & $3+, 8 / 12(66.7 \%)$ \\
\hline 21 & ${ }^{\#} 31 \mathrm{M}, \mathrm{AA}$ & 9.00 & 5.0 & $3+, 100 \%(300)$ & $3,3 / 10(30 \%)$ \\
\hline
\end{tabular}

$A A$ African American, $C$ Caucasian American, $L$ Latino American, $F$ female, $M$ male, $F S G S$ focal segmental glomerulosclerosis, $P T$ proximal tubules

*Human immunodeficiency virus (HIV) infection

\#Coronavirus disease 19 (COVID-19)
The levels of sCr in negative controls (mean: $0.95 \mathrm{mg} / \mathrm{dl}$ ) and FSGS group (mean: $1.57 \mathrm{mg} / \mathrm{dl}$ ) were significantly lower than that of collapsing glomerulopathy group (mean: $5.15 \mathrm{mg} / \mathrm{dl}$ ) (Table 1). Proteinuria data were not available for the negative controls as their kidneys were sampled for renal tumors. Mean protein/creatinine ratio in the FSGS group (mean: 6.46) reached nephrotic range proteinuria, but was still significantly lower than that in the collapsing glomerulopathy group (mean: 10.73). Individual data from FSGS and collapsing glomerulopathy cases were listed in Table 2. 


\section{CD133 staining highlighted hyperplastic podocytes in collapsing glomerulopathy}

Negative controls revealed positive CD133 staining in parietal epithelial cells without staining in the podocytes (Fig. 1A). Most FSGS biopsies also only showed positive CD133 in parietal epithelium (Fig. 1B). Occasionally, small clusters of hyperplastic podocytes were highlighted by CD133 staining; this observation was noted in 7 out of 15 FSGS cases (Fig. 1C). The striking finding was that all collapsing glomerulopathy biopsies showed positive staining in the clusters of proliferative podocytes (ranging from 14 to $81 \%$ of glomeruli), which displayed a mosaic pattern intermingled with collapsed glomerular capillary loops (Fig. 1 D-F). On dual CD133 and PAS-stained sections, negative control revealed normal glomerular capillary loops that stained positively for PAS (pinkish PAS staining), while CD133 staining (brownish) was positive in the parietal epithelial cells along the Bowman's capsule but not in the podocytes (Fig. 2A). Small hyperplastic podocytes were positive for CD133 in a few of the FSGS cases (Fig. 2B). In contrast, CD133 staining highlighted the florid podocyte hyperplasia surrounding the collapsed PAS-positive glomerular capillary loops of the collapsing glomerulopathy biopsies (Fig. 2 C, D). It was noted that CD133-positive hyperplastic podocytes were located outside of PAS-stained glomerular basement membranes while endocapillary endothelial cells and mesangial cells stained negatively for CD133 and were located within PAS-stained glomerular basement membranes. Electron microscopy revealed diffuse fusion of foot processes in both the primary FSGS group and the collapsing glomerulopathy group, confirming the nature of FSGS and collapsing glomerulopathy. Statistically, the percent of glomeruli with CD133-positive hyperplastic podocytes in the collapsing glomerulopathy group (mean: $34.90 \%$ ) was significantly more than that in the FSGS group (mean: $8.98 \%$ ) (Table 1). The combined score of positive CD133 in glomeruli in the collapsing glomerulopathy group (mean: 93.31) was also significantly higher than that in the FSGS group (mean: 15.43). The CD133-positive hyperplastic podocytes from both FSGS and collapsing glomerulopathy groups were significantly associated with the proteinuria levels $(r$-value $=0.359 ; p$-value $=0.0316$ ).

To confirm proliferative and undifferentiated nature of hyperplastic podocytes in collapsing glomerulopathy, proliferative marker Ki-67 and podocyte marker WT-1 were stained in negative control and collapsing glomerulopathy cases. Negative control glomeruli demonstrated no hyperplastic podocytes on routine hematoxylin/eosin-stained section, negative staining for CD133 in podocytes, and negative staining for $\mathrm{Ki}-67$, but positive nuclear staining for WT1 in terminally differentiated podocytes (Fig. 3A-D). In
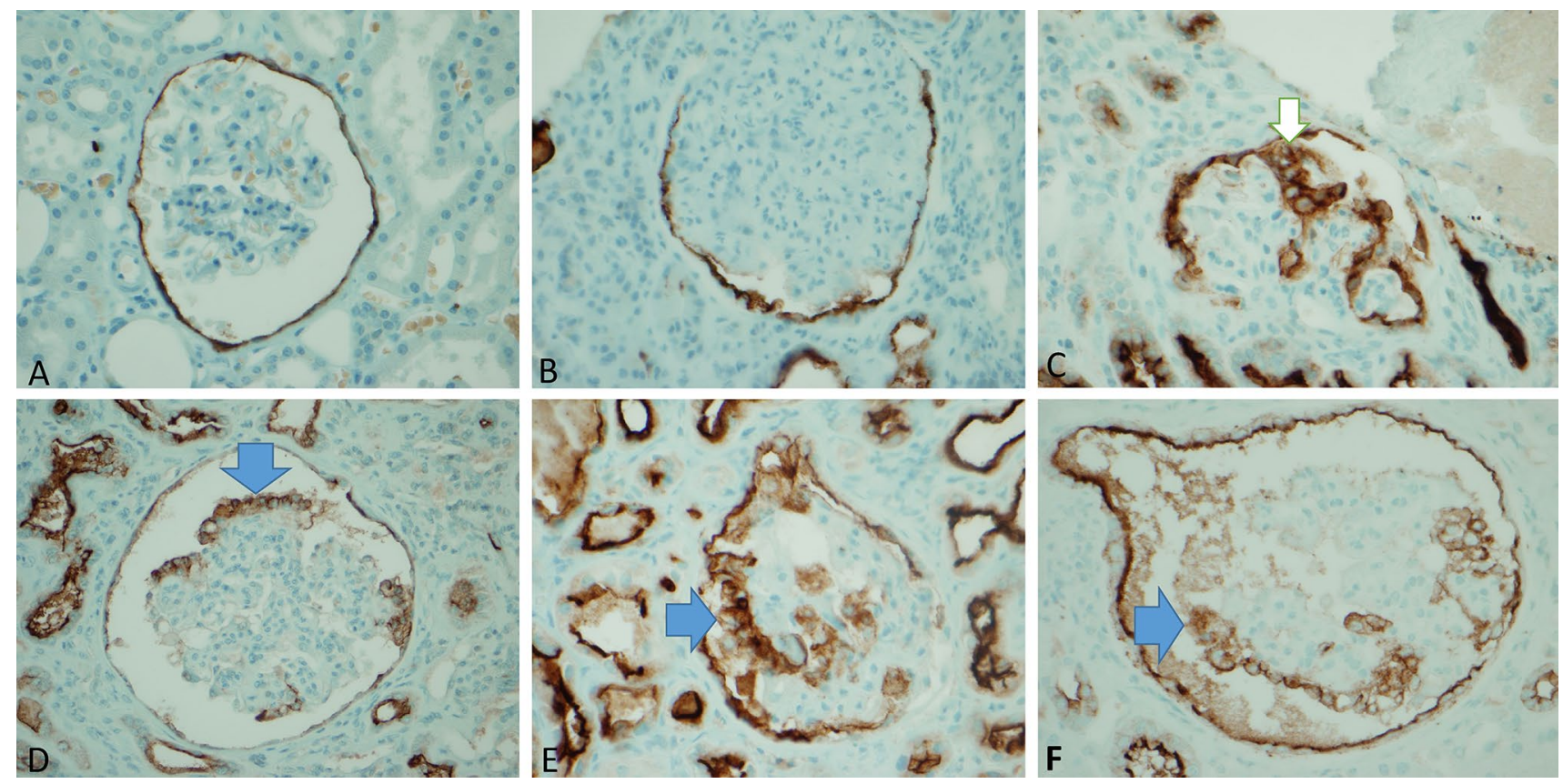

Fig. 1 CD133 expression in negative control, FSGS, and collapsing glomerulopathy. A CD133 was expressed in normal parietal epithelial cells along the Bowman's capsule but not in podocytes. B In most FSGS, parietal epithelial cells also stained positive for CD133, but podocytes were negative for this staining. C Occasionally, CD133 were positive in a small cluster of immature podocytes of FSGS (white arrow). In collapsing glomerulopathy (D, $\mathbf{E}$ and $\mathbf{F})$, there was a range of CD133-positive staining in the proliferative podocytes that was most characterized with a mosaic pattern intermingled with collapsed glomerular tufts (wide base blue arrows). Magnifications at $\times 600$ in $\mathbf{A}-\mathbf{F}$ 

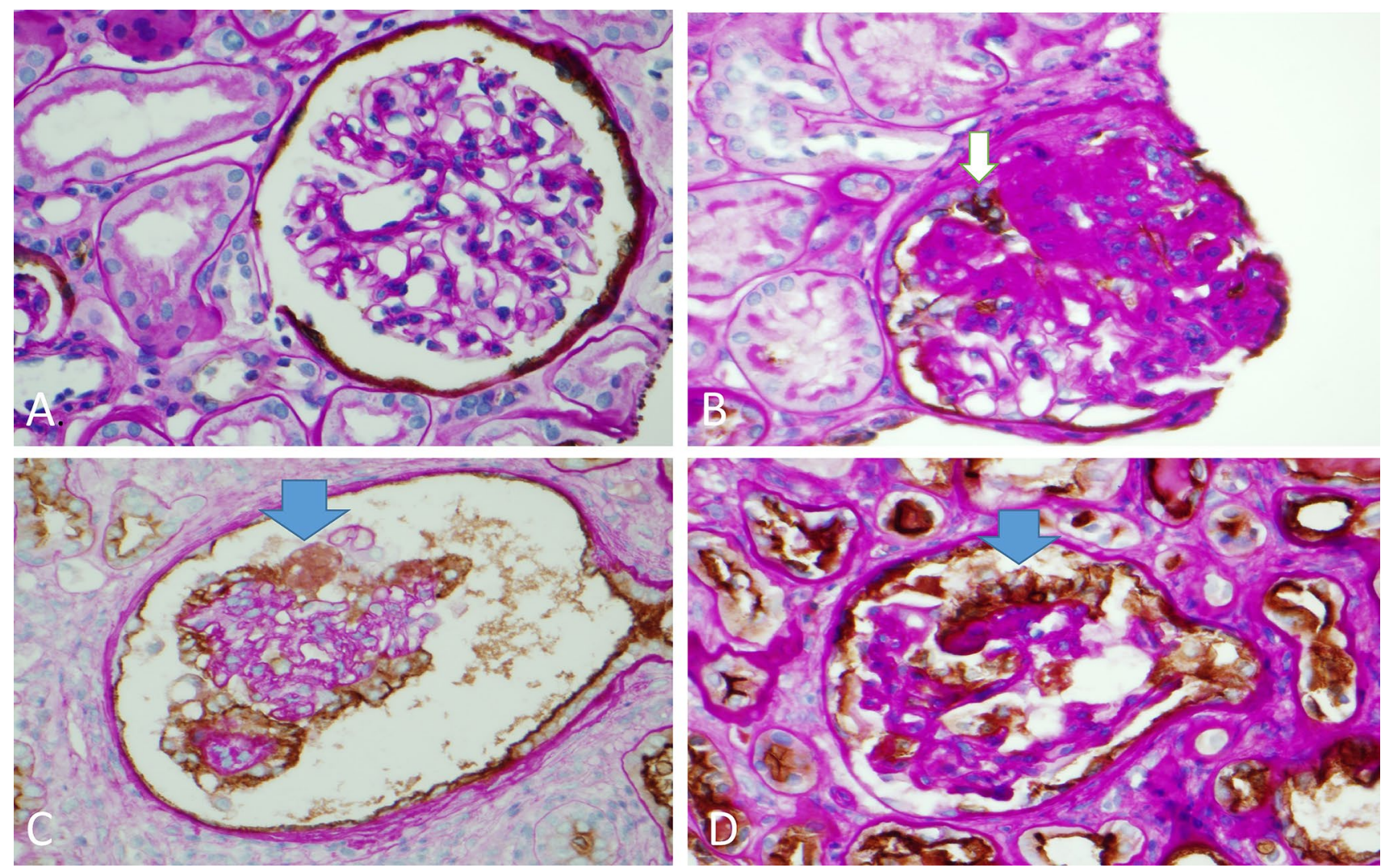

Fig. 2 CD133 and PAS dual stains in negative control, FSGS, and collapsing glomerulopathy. A PAS-stained delicate glomerular capillary loops with minimal mesangial cells. The parietal epithelium stained positively for CD133 while podocytes were negative for CD133 staining. B Occasional cluster of CD133-positive immature

podocytes was seen in a few FSGS cases (white arrow). In collapsing glomerulopathy, CD133 highlighted florid immature podocytes in C and $\mathbf{D}$ (indicated by wide base blue arrows). Magnifications at $\times 600$ in $\mathbf{A}-\mathbf{D}$

constract, the hyperplastic podocytes of collapsing glomerulopathy were identified on routine hematoxylin/eosin-stained sections (Fig. 3E and I), with positive CD133 staining (Fig. 3F and J), weakly positive staining for Ki-67 (Fig. 3G and K), but negative staining for WT-1 (Fig. 3H and L), indicating their proliferative but undifferentiated characteristics.

\section{CD133 staining confirmed early acute tubular injury and showed diffuse expressive pattern in collapsing glomerulopathy}

The negative controls revealed 1 or 2 CD133-positive progenitor cells per cross section of proximal tubules, which stained negatively for PAS, indicating that CD133-positive progenitor cells did not have reabsorption capacity (Fig. 4A). It was also interesting to see that the CD133-positive cells were actually located at the tip of the less curevature of the turning segements of the proximal tubules; the turning location was highlighted by PAS-positive tubular basement membranes with invaginations (Fig. 4A). At the early stage of acute tubular injury, there were side-by-side and groups of two or three

CD133-positive cells along proximal tubules, which were also negative for PAS staining (Fig. 4B). With various renal injury, there was a range of positive CD133 staining from 20 to $100 \%$ in proximal tubules, whereas PAS-positive brush borders were reduced proportionally in CD133-positive segments (Fig. 5B-D). For biopsies with collapsing glomerulopathy, the CD133 expression in the proximal tubules was strong $(3+)$ and diffuse, ranging from 60 to $100 \%$ (Fig. 5D). Furthermore, CD133 was found to be specific and sensitive in detecting acute tubular injury in correlation with serum creatinine, by showing an ROC area under the curve (AUC) value of 0.93 (very good category) (Fig. 6). CD133 combined scores of proximal tubules were significantly higher in the collapsing glomerulopathy group (mean: 252.38) when compared to the other two groups (mean: 0.83 in controls vs 56.67 in FSGS) (Table 1). 

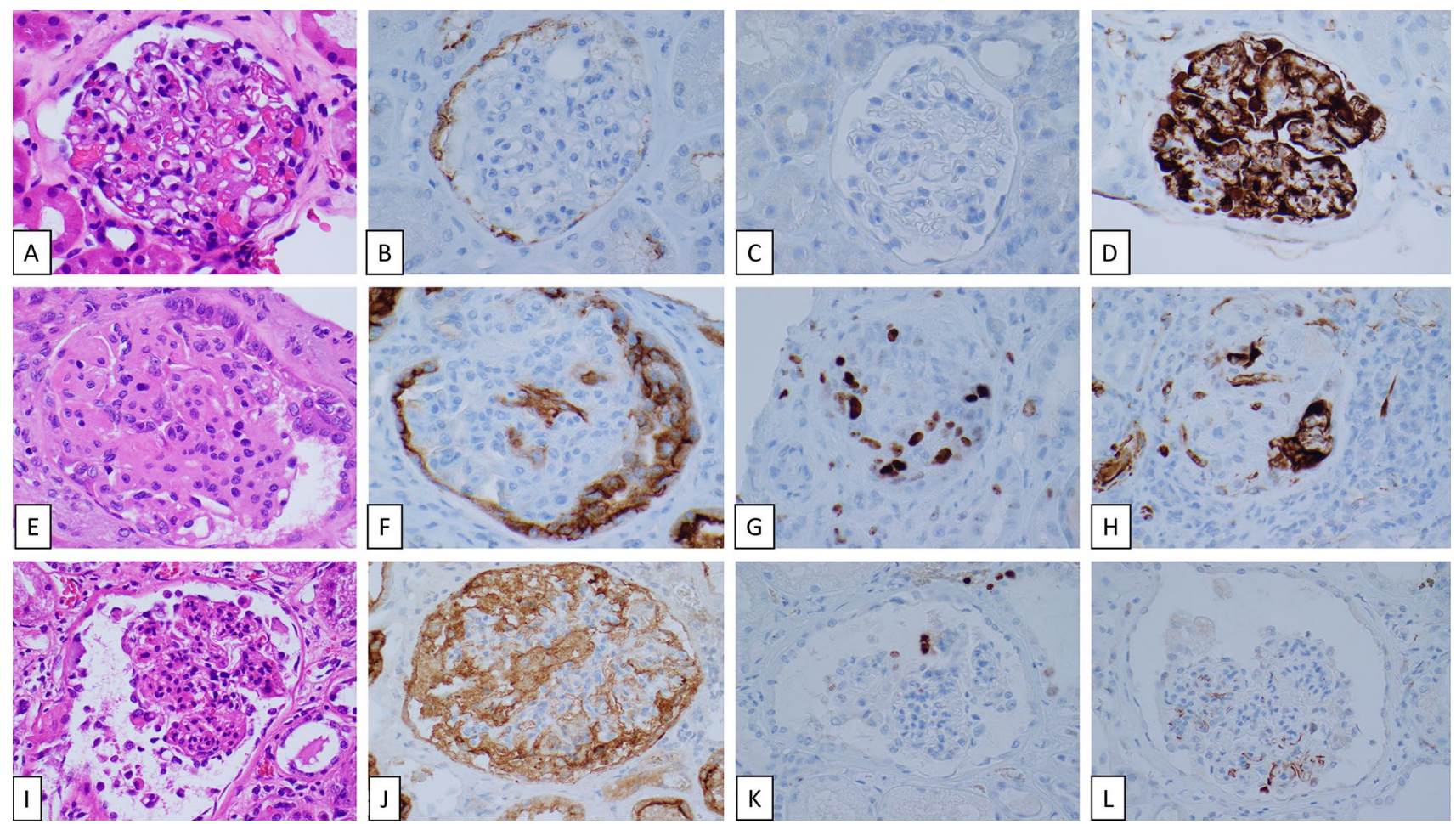

Fig. 3 Hematoxylin/eosin staining, and immune stains for Ki-67 (proliferative marker) and WT-1 (podocyte marker) in negative control glomeruli and glomeruli with hyperplastic podocytes. Negative control glomeruli with A hematoxylin/eosin staining, B negative Ki-67

staining and $\mathbf{C}$ positive WT-1 staining in podocytes. Hyperplastic podocytes with hematoxylin/eosin staining ( $\mathbf{D}$ and $\mathbf{G})$, positive Ki-67 staining (E and $\mathbf{H})$ and negative WT-1 staining (F and $\mathbf{I})$ from two collapsing glomerulopathy cases. Magnification $\times 600$ in $\mathbf{A}-\mathbf{L}$
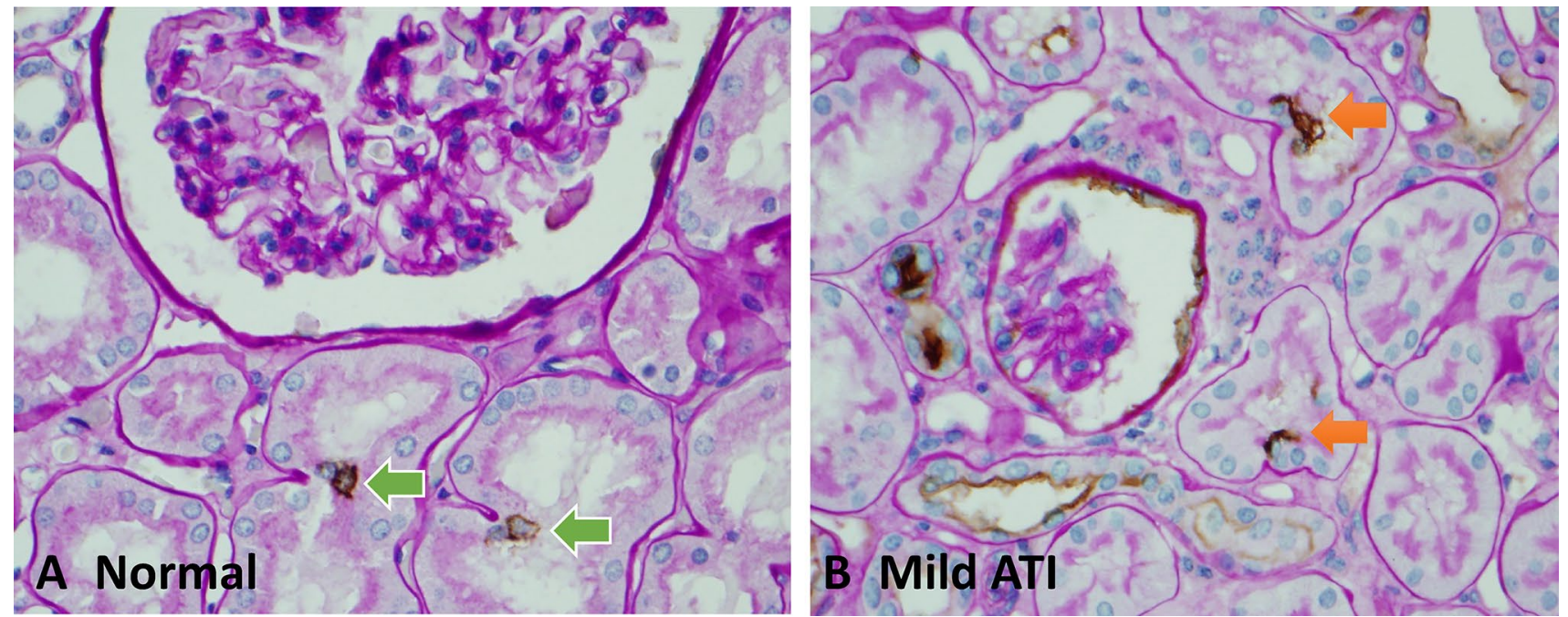

Fig. 4 CD133 in the normal proximal tubules and the proximal tubules with early tubular injury. A In normal proximal tubules, the CD133-positive progenitor cells were located at the niches of tubular turning segments (with PAS-positive tubular basement membranes showing invagination), which were not covered by PAS staining on their surfaces (green arrows). The remaining proximal tubular epithe-

lial cells were negative for CD133 staining but were covered by PASpositive brush borders. B At the early stage of acute tubular injury, CD133-positive cells extended the signals to the surrounding cells clustered in groups of two or three cells (orange arrows), which were also negative for dual-PAS staining on the surface. Magnifications at $\times 600$ for $\mathbf{A}, \mathbf{B}$ 

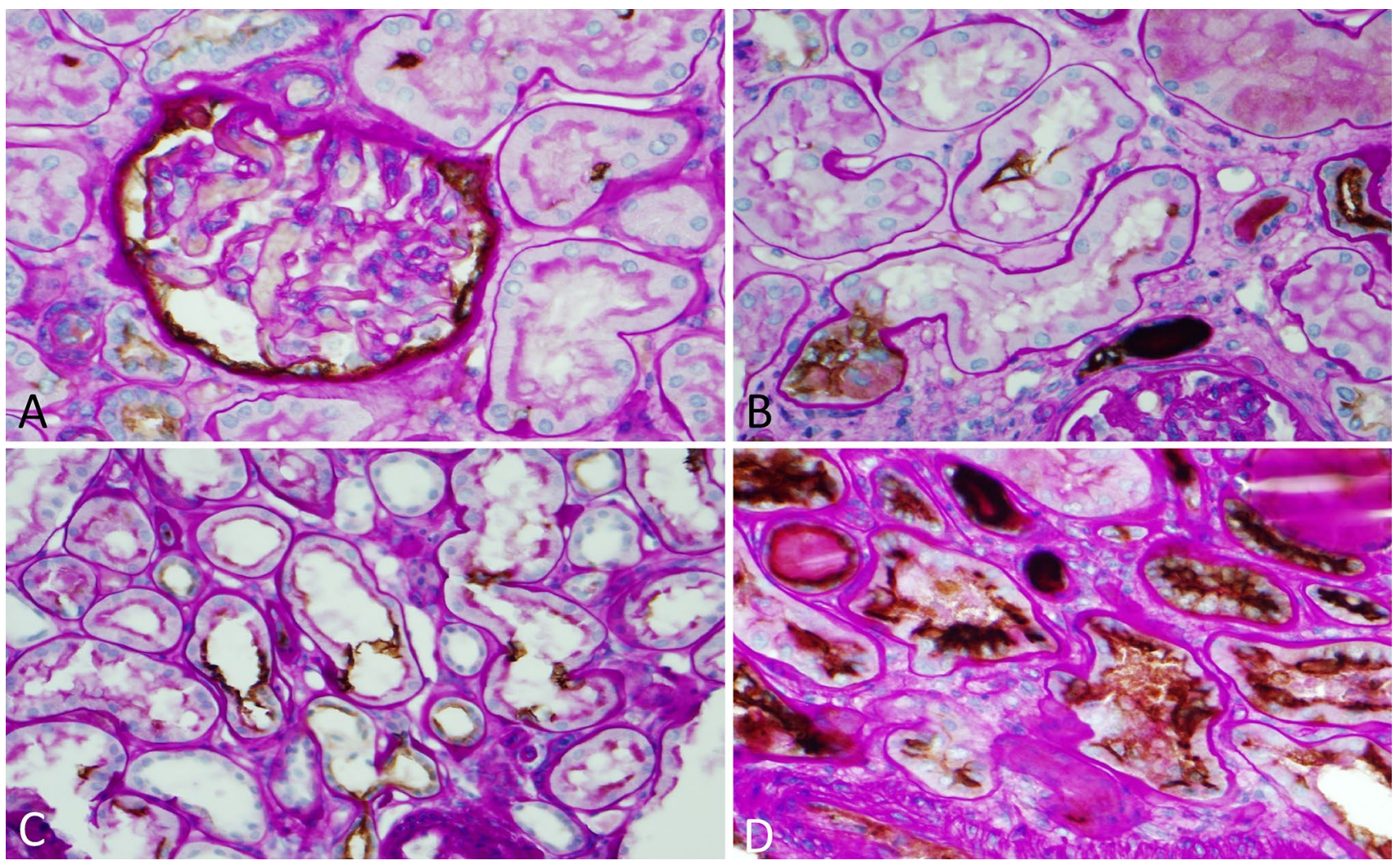

Fig. 5 Grading CD133 and PAS in acute tubular injury. A CD133 staining was graded as 0 in normal proximal tubules with intact brush border staining by PAS. B Mild scattered CD133 staining was graded as $1+$ with mild reduced PAS staining. C Darker CD133 staining

\section{Discussion}

Our data indicate that CD133-positive hyperplastic podocytes, located outside of PAS-positive glomerular basement membranes, were unique features in supporting the diagnosis of collapsing glomerulopathy. It should be noted that a few FSGS cases showed occasional CD133-positive podocytes as well. This indicates that there may be a small overlap between FSGS and collapsing glomerulopathy. Therefore, the activation of new podocyte production from the parietal epithelium can occur in any podocytopathy, although the proliferative activity of hyperplastic podocytes becomes more obvious in the cases of collapsing glomerulopathy. Smeets et al. have characterized this type of hyperplastic podocytes into $60 \%$ of immature podocytes $\left(\mathrm{CD} 133^{+}, \mathrm{CD}^{2} 4^{+}, \mathrm{PDX}^{-}\right.$, nestin $\left.{ }^{-}\right), 30 \%$ of mature podocytes $\left(\mathrm{CD} 133^{-}, \mathrm{CD}^{2} 4^{-}, \mathrm{PDX}^{+}\right.$, nestin $\left.^{+}\right)$and $10 \%$ of transitional podocytes $\left(\mathrm{CD} 133^{+}, \mathrm{CD} 24^{+}, \mathrm{PDX}^{-}\right.$, nestin $\left.{ }^{-}\right)$[18]. The current study showed that some of hyperplastic podocytes were positive for a proliferative marker $\mathrm{Ki}-67$, but negative for another podocyte marker WT-1 as shown previously [20], consistent with some proliferative but immature hyperplastic podocytes in the collapsing glomerulopathy $[18,20]$. The in more proximal tubules was graded as $2+$. D Strong and diffuse CD133 staining in a collapsing glomerulopathy was graded as $3+$. Magnifications at $\times 600$ for $\mathbf{A}-\mathbf{D}$

overall finding indicates that glomerular hyperplastic lesions are derived from the proliferation of parietal renal progenitors at different stages of their differentiation toward mature podocytes [18].

Smeets et al. also indicate that cellular crescents in crescentic glomerulonephritis and proliferative podocytes in collapsing glomerulopathy all come from the same parietal epithelium of the glomeruli [18]. Indeed, based on our stains from a previous study [11] and the current investigation, the biomarkers CD133, proliferative marker Ki-67 and WT-1 showed similar expressions in both cellular crescents and hyperplasic podocytes (Supplement Table 1), but both entities have different morphologic appearances and occur in different clinical scenarios. The cellular crescents are composed of more spindle-shaped epithelial cells, directly derived from random areas of the parietal epithelial cells along the Bowman's capsule and attached to the Bowman's capsule. Their aggregated crescentic cells can physically cause the collapse of glomerular capillary loops (Supplementary Fig. 1, the top half of schematic drawing). In contrast, the hyperplastic podocytes appear to follow the differentiation pathway from parietal epithelial cells to podocytes, look more cuboidal and are distributed along glomerular 


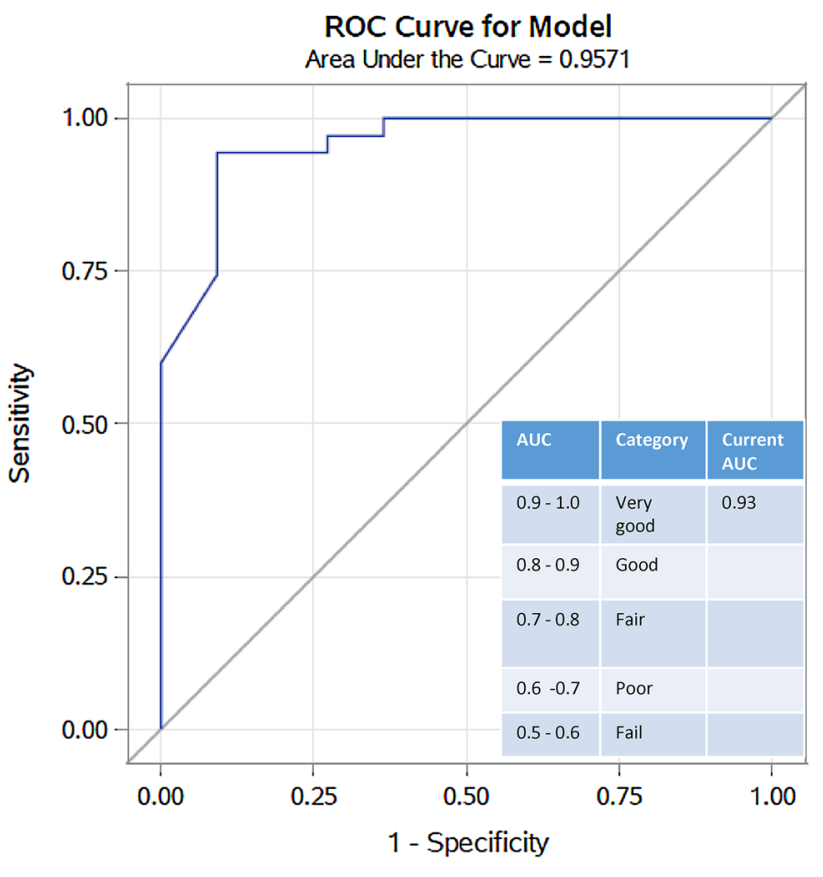

Fig. 6 Receiver operative characteristics (ROC) curve with analysis of area under the curve (AUC) for the performance of CD133 staining based on a range of serum creatinine levels in all three groups. The overall cases had an AUC of 0.93 (very good category)

capillary loops (Supplementary Fig. 1, the bottom half of schematic drawing). Clinically, cellular crescents occur mainly in primary crescent glomerulonephritis associated with either ANCA or anti-glomerular basement antibodies and these patients often present with acute renal failure and nephritic symptoms, hematuria and some proteinuria. In contrast, the hyperplastic podocytes are seen in the collapsing glomerulopathy and these patients often present with renal failure and severely nephrotic range of proteinuria.

From an embryology point of view, both parietal and visceral epithelial cells are derived from the cap mesenchyme in metanephors and form a double-layered network covering the glomerular capillary loops [21, 22]. Both podocytes and glomerular endothelium contribute to the building of glomerular basement membranes [23-25]. The podocytes interact with glomerular basement membranes through their foot processes and cellular cross-communication, which may play a key role in tethering and keeping the glomerular capillary loops open [26, 27]. During the development of collapsing glomerulopathy, the residual differentiated podocytes were reduced and partially replaced by clusters of immature hyperplastic podocytes in Bowman's space [28]. This may significantly reduce the role of podocytes in stretching the glomerular loops outward and hence reduce the crosscommunication and support to the glomerular endothelium. These factors would hence result in the collapse of glomerular capillary loops [25] (Supplementary Fig. 1, the bottom half of schematic drawing). In other words, the CD133-positive hyperplastic podocytes are immature podocytes that cannot carry out the normal function that only terminally differentiated (CD133-negative) podocytes can.

Common etiologies of collapsing glomerulopathy are HIV infection and mutation of APOL1 alleles in individuals of African American descent, but other infections and drug reactions can cause the disease as well [20, 29-31]. Since the beginning of 2020, severe acute respiratory syndrome coronavirus 2 (SARS-CoV-2) infected millions of people and caused many deaths worldwide. SARS-CoV-2 is known to cause coronavirus disease 19 (COVID-19) characterized by severe lung infection with subsequent infection of other organs through viral interaction with cell surface receptors (angiotensin-converting enzyme 2) [32-34]. Scattered studies using immunofluorescent staining and electron microscopy have reported direct renal infection in patients with COVID-19, causing acute tubular injury and collapsing glomerulopathy $[15,35,36]$. But several recent studies using RNA in-situ hybridization and electron microscopy report no definite SARS-CoV-2 detected in the renal biopsies or autopsy kidneys from COVID-19-positive patients $[16,17,37,38]$. All of our glomerulopathy patients with collapsing glomerulopathy were of African American descent. Although our study did not conduct genetic studies for the African American patients with collapsing glomerulopathy, previous studies have demonstrated that nearly all patients with collapsing glomerulopathy are from African descent and posses genetic vulnerability for APOL1 mutation, regardless of viral infection status [16, 29, 39].

Our second finding is that the CD133-positive progenitor cells were scattered along the proximal tubules, mostly seated at the tip of the lesser curvature of the turning proximal tubules (CD133 staining in Fig. 4A and a schematic illustration in Fig. 7A), similar to what has been reported by Lindgren et al. in 2011 [10]. They have also documented that the CD133 + tubular progenitor cells are co-localized with several biomarkers including vimentin, cytokeratin-7, and bcl-2 [10]. In this study, we found that CD133 + tubular progenitor cells stained negatively for PAS, indicating that they did not have the brush borders, thus most likely lacking of a reabsorption capacity. Taken together with the co-localization stains done by Lindgren et al. [10], the overall findings suggest that the CD133 + tubular progenitor cells may represent a second population of cells along the proximal tubular epithelium. It is well known that HIFs are the master transcription factors regulating hypoxia-associated transcription response [40, 41]. With normal oxygen, HIF- $1 \alpha$ is degraded. In contrast, hypoxia results in a retention of HIF- $1 \alpha$ that subsequently activates many genes, such as erythropoietin and CD133, to deal with the hypoxic challenge $[3,4,40]$. Therefore, we raise a possibility that the $\mathrm{CD} 133$ + progenitor cells along proximal tubules act either as "watch dogs" or 


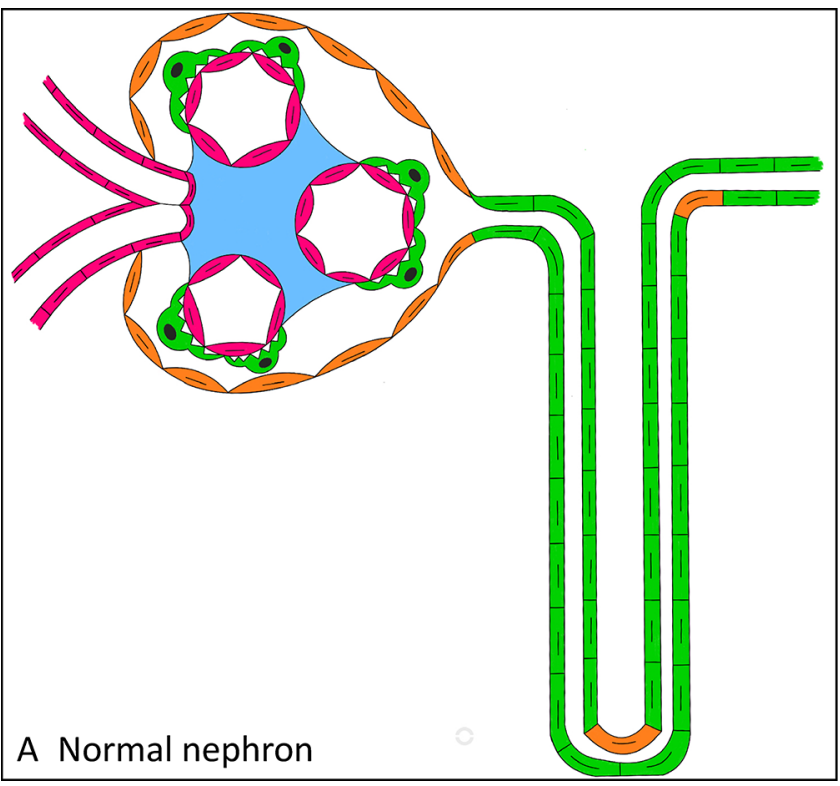

Fig. 7 Schematic comparison of normal nephron and collapsing glomerulopathy. A Normal glomerulus have orange-colored CD133positive parietal epithelial cells with CD133-negative podocytes and scattered CD133-positive progenitor cells at the tip of the less curvature of the turning proximal tubules. B In collapsing glomerulopathy,

"oxygen sensing receptor cells" and may represent a part of the HIF-associated hypoxic sensing and signaling mechanism. With early tubular injury, we identified that there were clusters of CD133-positive tubular cells in the absence of PAS staining. This implies that CD133-positive tubular progenitor cells, as potential "oxygen sensing receptor cells", may spread their injury signals to the surrounding epithelial cells first before they could spread their injury signals to the rest of the tubular epithelium.

Over a range of acute tubular injury, the staining for CD133 has provided us with a sensitive and specific tool to detect the acute tubular injury along proximal tubules based on the ROC curve presented. The mechanism of repairing proximal tubular injury has been debatable. In recent years, the theories for tubular repair mainly include two possibilities. The first theory is that the surviving tubular epithelial cells would repair the injured tubular epithelium [42]. The second theory would be that intraepithelial CD133-positive stem/progenitor cells can perform the repair [10-12]. Although both theories can play their roles in different stages of acute tubular injury, our observations indicate that stem/progenitor cells can repair tubular injury at least during the early stages of proximal tubular injury. CD133 can also be used to identify acute tubular injury of proximal tubules in any renal biopsies such as some FSGS cases in the primary FSGS group. However, the diffuse and strong expression of CD133 was the other unique feature in biopsies with collapsing glomerulopathy

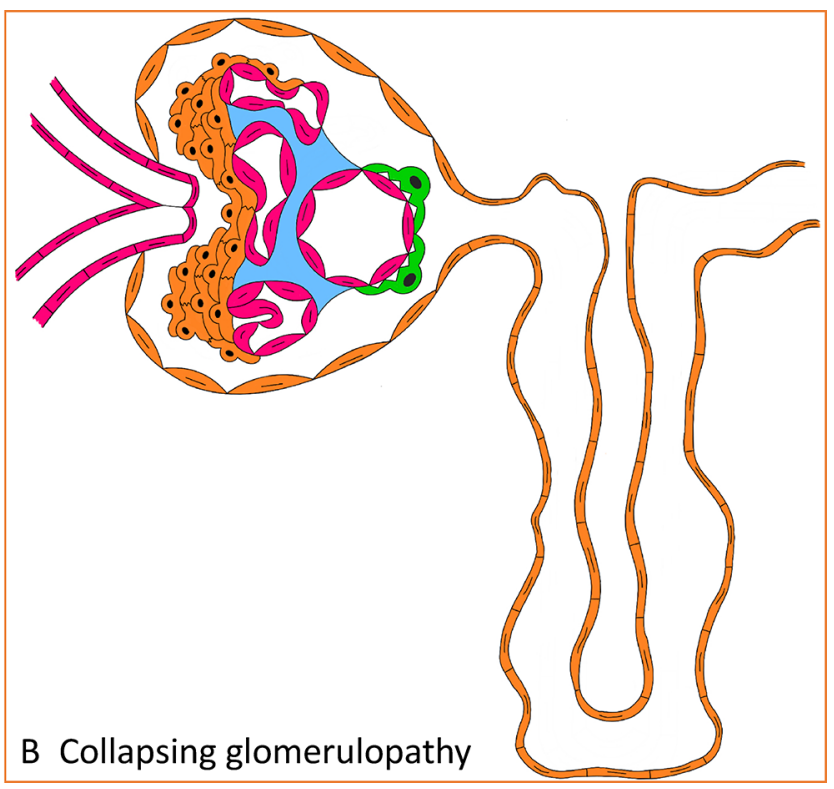

the orange-colored CD133-positive podocytes are clustered outside of the collapsed glomerular tufts and CD133 staining also shows diffuse expression in dilated and flat proximal tubular epithelial cells, indicating severe tubular injury

and correlated with severe acute renal failure in the vast majority of this renal disease. Both groups of FSGS and collapsing glomerulopathy had high levels of proteinuria but much less acute tubular injury was seen in FSGS group. Therefore, the severe acute tubular injury in the collapsing glomerulopathy group most likely resulted from collapsing loops associated ischemic insults to the proximal tubules rather than proteinuria alone.

In summary, a normal nephron has CD133-positive parietal epithelial cells and scattered CD133-positive progenitor cells along the proximal tubules (Fig. 7A). The dual features of CD133 expression in hyperplastic podocytes and injured proximal tubules can support a final diagnosis of collapsing glomerulopathy (Fig. 7B), particularly when hyperplastic podocytes are not florid and tubular cystic dilation is not obvious. CD133-positive hyperplastic podocytes may represent a common phenomenon seen over a spectrum of podocytopathies from FSGS to collapsing glomerulopathy in adults $[18,19]$. Based on the CD133 staining patterns in both immature podocytes and proximal tubules, we suspect that some FSGS cases, such as case \#9 in the FSGS group (Table 2) can be re-classified as a collapsing glomerulopathy. In collapsing glomerulopathy, the florid magnitude of immature hyperplastic podocytes (positively stained for CD133) appears to be an indirect index that reversely reflects sharply reduced functional podocytes (negative CD133 staining). The decrease in functional podocytes may be the key event that results in collapsed glomerular capillary loops 
and subsequently ischemic injury to the proximal tubules (diffuse positive CD133 staining).

Supplementary Information The online version contains supplementary material available at https://doi.org/10.1007/s11255-021-03078-0.

Acknowledgements Authors thank technologists in Beaumont histology laboratory and immuno-laboratory, Royal Oak, MI, for their excellent technical assistance.

Funding No funding was received for this study.

\section{Declarations}

Conflict of interest AYX declares that he has no conflict of interest. HDK declares that he has no conflict of interest. ZL declares that he has no conflict of interest. WL declares that she has no conflict of interest. PLZ declares that he has no conflict of interest.

Ethical approval This is a retrospective study using human renal biopsies, which study protocol has been approved by the Institutional Research Board of Beaumont Health, Michigan. This article does not contain any studies with animals performed by any of the authors.

Informed consent No informed consent is needed for this retrospective investigation with minimal risk, based on the IRB approval.

\section{References}

1. Katoh Y, Katoh M (2007) Comparative genomics on PROM1 gene encoding stem cell marker CD133. Int J Mol Med 19(6):967-970

2. Mizrak D, Brittan M, Alison M (2008) CD133: molecule of the moment. J Pathol 214(1):3-9

3. Li Z (2013) CD133: a stem cell biomarker and beyond. Exp Hematol Oncol 2(1):17

4. Liu H, Liu C, Qu Y (2021) The effect and molecular mechanism of hypoxia on proliferation and apoptosis of CD133+ renal stem cells. Bosn J Basic Med Sci 21(3):313-322

5. Sagrinati C, Netti GS, Mazzinghi B, Lazzeri E, Liotta F, Frosali F, Ronconi E, Meini C, Gacci M, Squecco R, Carini M, Gesualdo L, Francini F, Maggi E, Annunziato F, Lasagni L, Serio M, Romagnani S, Romagnani P (2006) Isolation and characterization of multipotent progenitor cells from the Bowman's capsule of adult human kidneys. J Am Soc Nephrol 17(9):2443-2456

6. Lazzeri E, Crescioli C, Ronconi E, Mazzinghi B, Sagrinati C, Netti GS, Angelotti ML, Parente E, Ballerini L, Cosmi L, Maggi L, Gesualdo L, Rotondi M, Annunziato F, Maggi E, Lasagni L, Serio M, Romagnani S, Vannelli GB, Romagnani P (2007) Regenerative potential of embryonic renal multipotent progenitors in acute renal failure. J Am Soc Nephrol 18(12):3128-3138

7. Ronconi E, Sagrinati C, Angelotti ML, Lazzeri E, Mazzinghi B, Ballerini L, Parente E, Becherucci F, Gacci M, Carini M, Maggi E, Serio M, Vannelli GB, Lasagni L, Romagnani S, Romagnani P (2009) Regeneration of glomerular podocytes by human renal progenitors. J Am Soc Nephrol 20(2):322-332

8. Appel D, Kershaw DB, Smeets B, Yuan G, Fuss A, Frye B, Elger M, Kriz W, Floege J, Moeller MJ (2009) Recruitment of podocytes from glomerular parietal epithelial cells. J Am Soc Nephrol 20(2):333-343

9. Smeets B, Uhlig S, Fuss A, Mooren F, Wetzels JF, Floege J, Moeller MJ (2009) Tracing the origin of glomerular extracapillary lesions from parietal epithelial cells. J Am Soc Nephrol 20(12):2604-2615

10. Lindgren D, Bostrom AK, Nilsson K, Hansson J, Sjolund J, Moller C, Jirstrom K, Nilsson E, Landberg G, Axelson H, Johansson ME (2011) Isolation and characterization of progenitor-like cells from human renal proximal tubules. Am J Pathol 178(2):828-837

11. Zhang PL, Hafron JM (2014) Progenitor/stem cells in renal regeneration and mass lesions. Int Urol Nephrol 46(11):2227-2236

12. Romagnani P (2011) Family portrait: renal progenitor of Bowman's capsule and its tubular brothers. Am J Pathol 178(2):490-493

13. Rednor SJ, Ross MJ (2018) Molecular mechanisms of injury in HIV-associated nephropathy. Front Med (Lausanne) 5:177

14. Kasembeli AN, Duarte R, Ramsay M, Mosiane P, Dickens C, Dix-Peek T, Limou S, Sezgin E, Nelson GW, Fogo AB, Goetsch S, Kopp JB, Winkler CA, Naicker S (2015) APOL1 risk variants are strongly associated with HIV-associated nephropathy in Black South Africans. J Am Soc Nephrol 26(11):2882-2890

15. Kissling S, Rotman S, Gerber C, Halfron M, Lamoth F, Sadallah S, Fakhouri F (2020) Collapsing glomerulopathy in a COVID-19 patient. Kidney Int. https://doi.org/10.1016/j.kint.2020.04.006

16. Wu H, Larsen CP, Hernandez-Arroyo CF, Mohamed MMB, Caza T, Sharshir M, Chughtai A, Xie L, Gimenez JM, Sandow TA, Lusco MA, Yang H, Acheampong E, Rosales IA, Colvin RB, Fogo AB, Velez JCQ (2020) AKI and collapsing glomerulopathy associated with COVID-19 and APOL 1 high-risk genotype. J Am Soc Nephrol 31(8):1688-1695

17. Kudose S, Batal I, Santoriello D, Xu K, Barasch J, Peleg Y, Canetta P, Ratner LE, Marasa M, Gharavi AG, Stokes MB, Markowitz GS, D'Agati VD (2020) Kidney biopsy findings in patients with COVID-19. J Am Soc Nephrol 31(9):1959-1968

18. Smeets B, Angelotti ML, Rizzo P, Dijkman H, Lazzeri E, Mooren F, Ballerini L, Parente E, Sagrinati C, Mazzinghi B, Ronconi E, Becherucci F, Benigni A, Steenbergen E, Lasagni L, Remuzzi G, Wetzels J, Romagnani P (2009) Renal progenitor cells contribute to hyperplastic lesions of podocytopathies and crescentic glomerulonephritis. J Am Soc Nephrol 20(12):2593-2603

19. Kopp JB, Anders HJ, Susztak K, Podesta MA, Remuzzi G, Hildebrandt F, Romagnani P (2020) Podocytopathies. Nat Rev Dis Prim 6(1):68

20. Barisoni L, Kriz W, Mundel P, D’Agati V (1999) The dysregulated podocyte phenotype: a novel concept in the pathogenesis of collapsing idiopathic focal segmental glomerulosclerosis and HIV-associated nephropathy. J Am Soc Nephrol 10(1):51-61

21. Ryan D, Sutherland MR, Flores TJ, Kent AL, Dahlstrom JE, Puelles VG, Bertram JF, McMahon AP, Little MH, Moore L, Black MJ (2018) Development of the human fetal kidney from mid to late gestation in male and female infants. EBioMedicine 27:275-283

22. Smyth IM, Caullen-McEwen LA, Caruana G, Black MJ, Bertram JF (2017) Development of the kidney: morphology and mechanisms. Fetal Neonatal Physiol 2(99):953-964

23. Abrahamson DR (2012) Role of the podocyte (and glomerular endothelium) in building the GBM. Semin Nephrol 32(4):342-349

24. Menon MC, Chuang PY, He CJ (2012) The glomerular filtration barrier: components and crosstalk. Int J Nephrol. https://doi.org/ $10.1155 / 2012 / 749010$

25. Nagata M (2016) Podocyte injury and its consequences. Kidney Int 89(6):1221-1230

26. Mundel P, Kriz W (1995) Structure and function of podocytes: an update. Anat Embryol (Berl) 192(5):385-397

27. Garg P (2018) A review of podocyte biology. Am J Nephrol 47(Suppl 1):3-13

28. Wiggins RC (2007) The spectrum of podocytopathies: a unifying view of glomerular diseases. Kidney Int 71(12):1205-1214 
29. Abid Q, Best Rocha A, Larsen CP, Schulert G, Marsh R, Yasin S, Patty-Resk C, Valentini RP, Adams M, Baracco R (2020) APOL1associated collapsing focal segmental glomerulosclerosis in a patient with Stimulator of Interferon Genes (STING)-Associated Vasculopathy with Onset in Infancy (SAVI). Am J Kidney Dis 75(2):287-290

30. Neyra JA, Vaidya OU, Hendricks A, Sambandam KK (2014) Collapsing focal segmental glomerulosclerosis resulting from a single dose of zoledronate. Nephron Extra 4(3):168-174

31. Patel AM, Zenenberg RD, Goldberg RJ (2018) De novo CMVassociated collapsing focal segmental glomerulosclerosis in a kidney transplant recipient. Transpl Infect Dis 20(3):e12884

32. Luan J, Lu Y, Jin X, Zhang L (2020) Spike protein recognition of mammalian ACE2 predicts the host range and an optimized ACE2 for SARS-CoV-2 infection. Biochem Biophys Res Commun 526(1):165-169

33. Zhou P, Yang XL, Wang XG, Hu B, Zhang L, Zhang W, Si HR, Zhu Y, Li B, Huang CL, Chen HD, Chen J, Luo Y, Guo H, Jiang RD, Liu MQ, Chen Y, Shen XR, Wang X, Zheng XS, Zhao K, Chen QJ, Deng F, Liu LL, Yan B, Zhan FX, Wang YY, Xiao GF, Shi ZL (2020) A pneumonia outbreak associated with a new coronavirus of probable bat origin. Nature 579(7798):270-273

34. Tan YJ, Teng E, Shen S, Tan TH, Goh PY, Fielding BC, Ooi EE, Tan HC, Lim SG, Hong W (2004) A novel severe acute respiratory syndrome coronavirus protein, U274, is transported to the cell surface and undergoes endocytosis. J Virol 78(13):6723-6734

35. Su H, Yang M, Wan C, Yi LX, Tang F, Zhu HY, Yi F, Yang HC, Fogo AB, Nie X, Zhang C (2020) Renal histopathological analysis of 26 postmortem findings of patients with COVID-19 in China. Kidney Int 98(1):219-227
36. Farkash EA, Wilson AM, Jentzen JM (2020) Ultrastructural evidence for direct renal infection with SARS-CoV-2. J Am Soc Nephrol 31(8):1683-1687

37. Sharma P, Uppal NN, Wanchoo R, Shah HH, Yang Y, Parikh R, Khanin Y, Madireddy V, Larsen CP, Jhaveri KD, Bijol V, Northwell C-RC (2020) Nephrology, COVID-19-associated kidney injury: a case series of kidney biopsy findings. J Am Soc Nephrol 31(9): 1948-1958

38. Santoriello D, Khairallah P, Bomback AS, Xu K, Kudose S, Batal I, Barasch J, Radhakrishnan J, D’Agati V, Markowitz G (2020) Postmortem kidney pathology findings in patients with COVID19. J Am Soc Nephrol 31(9):2158-2167

39. Friedman DJ, Pollak MR (2021) APOL1 nephropathy: from genetics to clinical applications. Clin J Am Soc Nephrol 16(2):294-303

40. Schodel J, Ratcliffe PJ (2019) Mechanisms of hypoxia signalling: new implications for nephrology. Nat Rev Nephrol 15(10):641-659

41. Shu S, Wang Y, Zheng M, Liu Z, Cai J, Tang C, Dong Z (2019) Hypoxia and hypoxia-inducible factors in kidney injury and repair. Cells 8(3):207

42. Humphreys BD, Valerius MT, Kobayashi A, Mugford JW, Soeung S, Duffield JS, McMahon AP, Bonventre JV (2008) Intrinsic epithelial cells repair the kidney after injury. Cell Stem Cell 2(3):284-291

Publisher's Note Springer Nature remains neutral with regard to jurisdictional claims in published maps and institutional affiliations. 